The authors reported no conflicts of interest.

The Journal policy requires editors and reviewers to disclose conflicts of interest and to decline handling or reviewing manuscripts for which they may have a conflict of interest. The editors and reviewers of this article have no conflicts of interest.

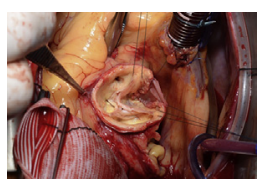

\section{REPLY: AORTIC ROOT REPAIR-ALL OR NOTHING}

\section{Reply to the Editor:}

With great interest, we read the letter of Guo and colleagues. ${ }^{1}$ The authors describe an alternative method for aortic root repair in acute type A aortic dissection using pericardial autograft. They suggest a significant reduction of remnant dissection tissue by removing the intimal dissection flap to the normal aortic wall near the annulus at the noncoronary sinus. Consequently, the intimal flap rim and adventitia near the coronary ostia are sutured to the pericardial patch using a running 5-0 PROLENE suture. Guo and colleagues assume that their technique reduces the risk of coronary stenosis due to blood flow and thrombus compression. Although this technique might reduce some dissected tissue (mainly of the noncoronary sinus), potential remnant dissection tissue around the coronary ostia remains. This diminishes the effort to reduce the risk of proximal bleeding and hence increase long-term durability. In contrast, the suggested technique is time-consuming while demanding experienced surgical skills and therefore less feasible for the unexperienced surgeon. The implementation of any pericardial patch for root repair may also lead to anatomic deformity and consequently aortic valve insufficiency.

Our institutional experience with pericardial patch for aortic root repair (for an exemplary picture, see Figure 1) underlines the missing additional (long-term) benefit of this method. Our technique (instead of Tanaka and colleagues $^{2}$ ) does not include horizontal mattress sutures while using a finer running suture (5-0 PROLENE), making it a fast approach while minimizing bleeding. However, the described technique from Guo and colleagues can be suitable for selected cases (ie, noncoronary sinus dilation).

In our opinion, aortic root repair should be an easily applicable and fast approach (even for the inexperienced surgeon), considering complexity and effectiveness true to the motto: less is sometimes more.

Arash Motekallemi, MD Angelo M. Dell'Aquila, MD

Andreas Rukosujew, MD

Department of Cardiothoracic Surgery

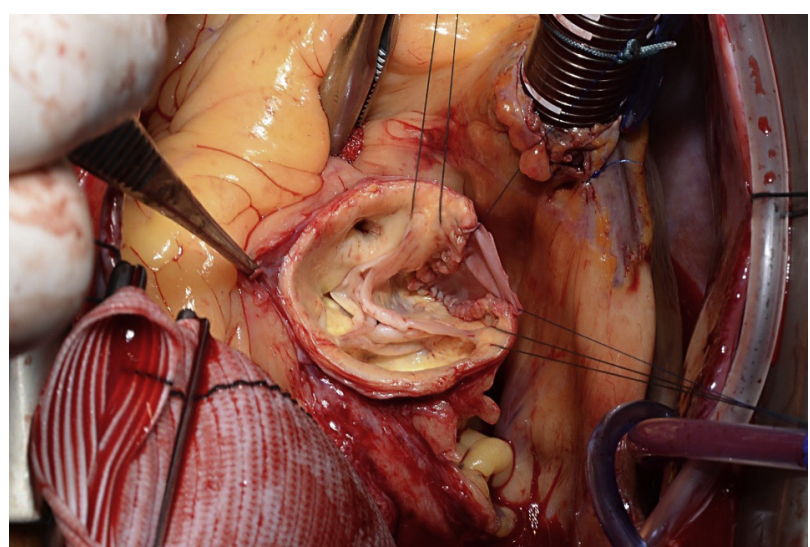

FIGURE 1. Aortic root repair with pericardial patch (Vascu-Guard; Baxter Healthcare, Munich, Germany) of the noncoronary sinus.

University Hospital Münster Münster, Germany

\section{References}

1. Guo H-W, Chang Y, Qian X-Y. Aortic root repair using pericardial autograft for acute type A aortic dissection. J Thorac Cardiovasc Surg. 2021;161:e154.

2. Tanaka K, Morioka K, Li W, Yamada N, Takamori A, Handa M, et al. Adventitial inversion technique without the aid of biologic glue or Teflon buttress for acute type A aortic dissection. Eur J Cardiothorac Surg. 2005;28:864-9.

https://doi.org/10.1016/j.jtcvs.2020.08.022

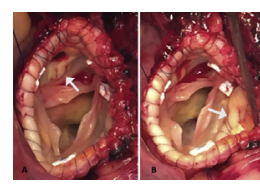

\section{REPLY FROM THE AUTHOR: RESPECT MOTHER NATURE- AORTIC ROOT REPAIR IN ACUTE TYPE A AORTIC DISSECTION Reply to the Editor:}

In their Letter to the Editor, Guo and colleagues ${ }^{1}$ describe their technique of aortic root repair or replacement with autologous pericardium in acute type A aortic dissection (ATAAD) in response to my group's previous study on direct repair of the aortic root without surgical adjuncts. ${ }^{2}$ Because of concerns of (1) bleeding from the proximal suture line, (2) long-term durability of the repaired root with dissected tissue, and (3) coronary stenosis, they replaced most dissection flaps at all 3 coronary sinuses with autologous pericardium. ${ }^{1}$ In their letter, Guo and colleagues $^{1}$ claim that their technique was safe and effective, although they do not describe the indications for this technique, nor do they report the short- or long-term outcomes.

I consider their technique to represent a partial aortic root replacement, because they replaced the dissection flap (more than two-thirds of the media of the aortic wall) of all 3 sinuses with pericardium. Should we replace the dissection flap of all 3 coronary sinuses for a 